\title{
Editorial
}

Nephrology

\section{Access to Kidney Transplantation: Is There a Potential Conflict of Interest?}

\author{
Mario F. Rubin Matthew R. Weir \\ Division of Nephrology, Department of Medicine, University of Maryland School of Medicine, Baltimore, Md., USA
}

Kidney transplantation has been demonstrated to be the optimal treatment for many, if not most, patients with end-stage renal disease (ESRD), providing both a better quality of life, as well as duration of life, compared to continuing dialysis as a method of treatment $[1,2]$. However, there exists a vast difference with regards to referral for kidney transplantation and kidney transplantation rates in the United States [3-5].

In the study by Gander et al. [6], it is quite evident that the dialysis facility transplant philosophy for referral varies considerably, and those dialysis centers whose staff have a more positive attitude toward transplantation demonstrate a much higher referral rate for kidney transplantation.

Prior work by these same authors [7] has demonstrated that factors associated with a lower standardized transplant ratio in the United States ESRD networks include a for-profit status of the dialysis facility, facilities with a higher percentage of African American or diabetic patients, as well as patients with no health insurance. On the other hand, dialysis facilities with a larger number of staff, ESRD network regions with a higher number of transplant centers, as well as facilities with more patients who were employed, or on peritoneal dialysis had higher standardized transplant ratios.

The observations of Gander et al. [6], although important, must be tempered by the understanding that their
(C) 2015 S. Karger AG, Basel

0250-8095/15/0416-0502\$39.50/0
ESRD network for this research effort (Network 6), which covers the southeastern region of the United States and serves the states of North Carolina, South Carolina, and Georgia, has the lowest standardized transplant ratio of all networks in the country. Thus, this study specifically examines the network that regulates the dialysis centers that were least likely to refer their patients for transplantation. It is therefore not surprising that fewer than $25 \%$ of these facilities reported transplantation as their first option of consideration. This low transplantation referral rate is likely related to the fact that this region serves more African-Americans who may have less health insurance and more socioeconomic disadvantage, and they may be cared for by for-profit dialysis centers.

The lower standardized transplant ratio in Network 6 may be less prevalent in other ESRD networks serving other demographic populations or may have more nonprofit dialysis facilities. Since profit depends, in part, on a larger number of patients in any center, it may not be surprising that dialysis staff who are paid by the centers, would be less likely to refer their patients for transplantation. Additionally, physician compensation for posttransplant care is far less than the capitated rate for supervising these patients on dialysis. Moreover, the time commitment for supervising immunosuppression and many of the medical comorbidities associated with posttransplantation care requires additional infrastructural 
support systems such as nurses and/or coordinators in order to help with the laboratory monitoring and medication level adjustments, which are so critical for the long-term success of transplantation. Thus, from an economic standpoint, despite its increased Medicare costs, dialysis remains a preferred option for many dialysis facilities and health care providers. The disparity in compensation relative to the increased work load required for managing kidney transplant patients likely plays a role in the decision-making process on referral for transplantation, especially for patients in for-profit units.

Some of the other issues identified by the observations of Gander et al. [6], relate to the fact that a majority of the respondents had different backgrounds. Fifty-one percent of the respondents were nurse managers, nearly $22 \%$ were social workers and the remaining was unspecified. There may be substantial differences in the perceptions for need for referral among these different respondent groups. Whether some of these respondent groups were more or less concerned about the profitable nature of the center of their employment may have played a role in their decision-making process on whether to refer for transplantation or not. However, the major point of this paper is well taken; there is something concerning about
ESRD Network 6 and their referral pattern for kidney transplantation, given the fact that kidney transplantation is associated with improved quality and quantity of life and is associated with reduced hospitalization and costs compared to dialysis [1,2]. More effort needs to be made to evaluate opportunities for improvement, particularly in the southeastern region of the United States. Recent reviews [8,9] have highlighted this problem and have made suggestions about how to improve access to care, especially for those patients with a socioeconomic disadvantage.

This study highlights the need to develop a national database with uniform transplant status codes that directly correlate with the different steps in the transplantation process. This may be helpful not only to study regional differences and referral patterns and transplantation barriers but also to develop interventions and ultimately assess their impact. Moreover, CMS needs to consider a more uniform policy for capitating and reimbursing the medical care of patients referred for transplantation so that there is parity with those receiving hemo- and peritoneal dialysis. This would remove some of the conflict of interests that currently exists with regards to referral patterns for transplantation, related to the profitable nature of the dialysis center, and individual health care providers.

\section{References}

1 Danovitch GM: Options for patient with kidney failure; in Danovitch GM (ed): Handbook of Kidney Transplantation, ed 2. Philadelphia, Lippincott Williams \& Wilkins, 2005, pp $1-22$.

2 Tonelli M, Wiebe N, Knoll G, et al: Systematic review: kidney transplantation compared with dialysis in clinically relevant outcomes. Am J Transplant 2011;11:20932109.

3 Axelrod DA, Dzebisashvili N, Schnitzler MA, et al: The interplay of socioeconomic status, distance to center, and interdonor service area travel on kidney transplant access and outcomes. Clin J Am Soc Nephrol 2010;5:22762288 .
4 Epstein AM, Ayanian JZ, Keogh JH, et al: Racial disparities in access to renal transplantation - clinically appropriate or due to underuse or overuse? N Engl J Med 2000;343:15371544, 2 p preceding 1537.

5 Johansen KL, Zhang R, Huang Y, Patzer RE, Kutner NG: Association of race and insurance type with delayed assessment for kidney transplantation among patients initiating dialysis in the United States. Clin J Am Soc Nephrol 2012;7:1490-1497.
6 Gander J, Browne T, Plantinga L, et al: Dialysis facility transplant philosophy and access to kidney transplantation in the Southeast. Am J Nephrol 2015;41:504-511.

7 Patzer RE, Plantinga L, Krisher J, Pastan SO Dialysis facility and network factors associated with low kidney transplantation rates among United States dialysis facilities. Am J Transplant 2014;14:1562-1572.

8 Patzer RE, Pastan SO: Kidney transplant access in the Southeast: view from the bottom. Am J Transplant 2014;14:1499-1505.

9 Srinivas TR: Kidney transplant access in the Southeastern United States: the need for a top-down transformation. Am J Transplant 2014;14:1506-1511. 